\title{
Long-Term Methamphetamine Administration in the Vervet Monkey Models Aspects of a Human Exposure: Brain Neurotoxicity and Behavioral Profiles
}

\author{
William P Melega*, 1,2, Matthew J Jorgensen², Goran Laćan', Baldwin M Way, Jamie Pham', \\ Grenvill Morton ${ }^{1}$, Arthur K Cho' ${ }^{1}$ and Lynn A Fairbanks ${ }^{2}$ \\ 'Department of Molecular and Medical Pharmacology, University of California, Los Angeles, CA, USA; ${ }^{2}$ Semel Institute for Neuroscience and \\ Behavior, David Geffen School of Medicine at University of California, Los Angeles, CA, USA; ${ }^{3}$ Department of Psychology, University of California, \\ Los Angeles, CA, USA
}

\begin{abstract}
Methamphetamine (METH)-associated alterations in the human striatal dopamine (DA) system have been identified with positron emission tomography (PET) imaging and post-mortem studies but have not been well correlated with behavioral changes or cumulative METH intake. Animal studies that model some aspects of human long-term METH abuse can establish dose-dependency profiles of both behavioral changes and potential brain neurotoxicities for identifying consequences of particular cumulative exposures. Based on parameters from human and our monkey pharmacokinetic studies, we modeled a prevalent human METH exposure of daily multiple doses in socially housed vervet monkeys. METH doses were escalated over 33 weeks, with final dosages resulting in estimated peak plasma METH concentrations of $\mathrm{I}-3 \mu \mathrm{M}$, a range measured in human abusers. With larger METH doses, progressive increases in abnormal behavior and decreases in social behavior were observed on 'injection' days. Anxiety increased on 'no injection' days while aggression decreased throughout the study. Thereafter, during 3 weeks abstinence, differences in baseline vs post-METH behaviors were not observed. Post-mortem analysis of METH brains showed $20 \%$ lower striatal DA content while autoradiography studies of precommissural striatum showed $35 \%$ lower $\left[{ }^{3} \mathrm{H}\right]$ WIN35428 binding to the DA transporter. No statistically significant changes were detected for $\left[{ }^{3} \mathrm{H}\right]$ dihydrotetrabenazine binding to the vesicular monoamine transporter (METH-lower by $10 \%$ ) or for $\left[{ }^{3} \mathrm{H}\right] \mathrm{SCH} 23390$ and $\left[{ }^{3} \mathrm{H}\right]$ raclopride binding to DA DI and D2 receptors, respectively. Collectively, this long-term, escalating dose METH exposure modeling a human abuse pattern, not associated with high-dose binges, resulted in dose-dependent behavioral effects and caused persistent changes in presynaptic striatal DA system integrity.

Neuropsychopharmacology (2008) 33, I44I - |452; doi:I0.1038/sj.npp. I 30 I 502; published online I I July 2007
\end{abstract}

Keywords: methamphetamine; dopamine; dopamine transporter; vesicular monoamine transporter; nonhuman primate

\section{INTRODUCTION}

As methamphetamine (METH) abuse becomes more widespread, increases in the number of METH-dependent individuals have been reported (Lineberry and Bostwick, 2006). Although behavioral consequences associated with long-term METH exposure have been extensively documented in METH abusers (Cretzmeyer et al, 2003; Ling et al, 2006; Meredith et al, 2005), the corresponding magnitude and duration of brain alterations remain unclear due, in part, to the paucity of relevant human data. The framework

* Correspondence: Dr WP Melega, Department of Molecular and Medical Pharmacology, David Geffen School of Medicine at UCLA, Box 951735, 28-117 BRI, Los Angeles, CA 90095-1735, USA, Tel: + I 310 206 1797, Fax: + I 310206 9934, E-mail:wmelega@mednet.ucla.edu Received 27 December 2006; revised 6 June 2007; accepted 6 June 2007 for postulating METH-associated brain changes in humans has been derived mainly from results of experimental METH studies, predominantly in rodents (Seiden, 1985, 1996; Bowyer and Holson, 1995; Davidson et al, 2001; Cadet et al, 2003) and relatively fewer in nonhuman primates (Fischman and Schuster, 1977; Melega et al, 1997; Madden et al, 2005). The extent to which those characterizations can be generalized for interpreting METH's effects on human brain neurochemistry has remained tenuous for many years.

Now, as was observed in animal studies, some METHassociated effects on the striatal dopamine (DA) system have also been consistently shown in humans, in vivo, with PET imaging, namely, lower dopamine transporter (DAT) ligand binding (15-30\%) (McCann et al, 1998; Sekine et al, 2001; Volkow et al, 2001; Wang et al, 2004; Johanson et al, 2006). A post-mortem study has also shown lower DAT ligand binding, in addition to reductions in DA and tyrosine 
hydroxylase protein content (Wilson et al, 1996). Collectively, these studies are extremely valuable in highlighting brain alterations associated with METH abuse but tend to generalize other distinguishing features of dosage, drug purity, frequency of administration, and cumulative exposure that may differentiate classes of METH behavioral and brain profiles. That is, a single profile of METH abuse may not represent the optimal conceptual framework for designing behavioral and pharmacological therapies for all METH abusers.

Evidence for a METH dose dependency underlying the extent of brain and behavioral alterations can be obtained through animal studies with defined dosage regimens. Accordingly, in this study, an escalating dose METH (EDMETH) administration in socially housed vervet monkeys was used to model some aspects of a human daily multiple dose METH exposure similar to that characterized in a study of 120 METH abusers in which the majority of individuals used METH 1-3 times per day and for more than 20 days per month (Simon et al, 2002). For modeling human plasma METH and amphetamine (AMPH) concentrations, pharmacokinetic studies were first conducted in the monkey to obtain kinetic rate parameters since METH half-life and metabolism differences between humans and animals can significantly affect overall exposure duration and interpretation of the resultant biochemical effects (Cho et al, 2001). Subsequently, we modeled a range of METH exposures by administering escalating doses that increased in frequency to 3 doses/day for 4 days/week. We hypothesized that such METH exposure would result in dose-dependent behavioral alterations that could be differentiated on 'injection' and 'no injection' observation days. Further, we anticipated that behavioral alterations would persist, albeit to a lesser extent, throughout a subsequent extended period of METH abstinence. Thereafter, brain analyses would document the extent of alterations in phenotypic markers associated with striatal DA and serotonin system integrity.

For this study, METH was administered to adult male vervet monkeys living in social groups containing a full range of different age/sex animals. The social housing was considered a critical component of the study design because it allowed for behavioral assessments of drug-related changes in affiliative and agonistic behaviors. Such measurements are highly relevant for behavioral models of the human condition, but are not possible to obtain in individually housed animals. Additionally, since the METH exposure extended over months and different seasons of the year, concurrent control groups were also considered necessary to account for potential time- and groupdependent behavioral changes. Without those control groups, behavioral changes that evolved independent of drug treatment could have been attributed to the METH exposure.

The post-mortem characterization of METH's effects after 3 weeks of abstinence was focused on a regional analysis of striatal DA system integrity for comparison with the results of human PET imaging studies of METH abusers who were scanned after several weeks to months of abstinence. With that correspondence, other consequences of long-term METH exposure in humans, unattainable from clinical studies, may then be acquired from more detailed analyses of the animal model. Further, upon identification of brain structures and behavioral functions altered by a particular METH exposure pattern, targeted pharmacological treatments can then be designed and evaluated before human applications.

\section{MATERIALS AND METHODS}

Vervet monkeys (Chlorocebus aethiops) were obtained from the UCLA/VA Vervet Research Colony in Sepulveda, CA and were assigned to either the METH pharmacokinetics or long-term METH exposure study. Animal care was in accordance with the National Research Council, Guide for the Care and Use of Laboratory Animals (1996), and approved by the Institutional Animal Care and Use Committee at the University of California, Los Angeles and the Greater Los Angeles Veterans Administration.

\section{Study 1: METH Pharmacokinetics Study}

Subjects. METH-naive male animals (age range: 5-7 years; $n=4)$ that were not included in the long-term METH study were transferred to UCLA and housed in individual cages for the duration of the study. The animals were habituated to sitting in a customized primate chair for 2 weeks before the pharmacokinetics study.

METH administration and experimental design. On the study day, the animal was placed in the chair and a catheter was inserted in the tail vein $30 \mathrm{~min}$ before injection of $0.2 \mathrm{mg} / \mathrm{kg} \quad S-(+)$-methamphetamine hydrochloride, i.m. (Sigma-Aldrich, St Louis, MO). Blood samples were obtained at $0.25,0.5,0.75,1,2$, and $4 \mathrm{~h}$. The animal was returned to its cage and blood samples were obtained at 6,8 , 10,12 , and $24 \mathrm{~h}$ from either the tail or femoral vein while temporarily restrained; the plasma fraction was frozen at $-80^{\circ} \mathrm{C}$ until analyzed.

Pharmacokinetic modeling. The pooled data representing all animals' blood samples were used to derive pharmacokinetic parameters for METH. The data were fitted to an open two-compartmental model with no lag time. The appearance of AMPH in the plasma following the METH dose was fitted to a one-compartment - first order appearance and elimination, no lag time model (WinNonlin, 3.0 program; Pharsight Corp, Mountain View, CA). An i.v. route of administration was used to model the METH plasma concentrations, based on the relatively rapid absorption following i.m. injection, ie the highest METH plasma concentration was measured at the first data point at $15 \mathrm{~min}$, followed by rapid decreases by the second data point at $30 \mathrm{~min}$. Metabolism-generated AMPH plasma concentrations were factored into the monkey model.

Pharmacokinetic parameters for the human METH exposure were obtained from prior human METH studies (Cook et al, 1993) and used in a one-compartment i.v. bolus, no lag time, first order elimination model. AMPH concentrations were not factored into the human profile insofar as metabolism-generated AMPH plasma concentrations are relatively low, being $10-20 \%$ that of METH (Cook et al, 1993). Additionally, modeling parameters for 
metabolism-generated AMPH could not be estimated since its rate constants have not been reported for humans.

\section{Study 2: Escalating Dose-METH Exposure}

Subjects and housing. The focal subjects in this study were 12 adult male vervet monkeys (age range 6.9-9.9 years, weight range $6.8-8.7 \mathrm{~kg}$ ) that were housed in three outdoor social groups at the joint UCLA/VA Vervet Research Colony. Each outdoor enclosure measured approximately $30 \mathrm{~m}^{2}$ and contained perches, play structures, swings, and other toys. All focal subjects were vasectomized before the beginning of the study.

The social groups were formed and allowed to habituate for approximately 3 months before study initiation. After focal subjects were randomly assigned to a group, they were randomly designated as either METH or Control subjects so as to reduce confounds with dominance rank effects on behavior: two METH and two Control focal subjects in each of three social groups concurrently. Each group also consisted of 3-4 adult females, 2-7 juveniles, and 0-3 infants, with no other adult males present throughout the study. One METH subject died of an unknown illness during the study. There was no evidence that experimental manipulation caused this death. Subjects were always housed within their social groups except during experimental manipulations.

Capture training and distress ratings. During the habituation period, focal subjects were trained to accept daily capture and injections (see Supplementary Information for details).

Experimental design. The study took place over a 1-year period. An initial 3-month habituation was followed by a 4-week pretest period (Baseline), 8 Dose periods (Dose 1-8) of 4-5 weeks each, and a 3-week abstinence (Post-test) period (Table 1).

Methamphetamine administration. METH subjects were administered $S-(+)$-methamphetamine hydrochloride, i.m.
(Sigma-Aldrich) dissolved in saline (volume of $0.3-0.4 \mathrm{ml}$ ) at the daily dose and frequency shown in Table 1. Control subjects received a comparable volume of saline at the same frequency as the METH subjects. Personnel performing the injections were blind to the condition of all subjects.

Behavioral observations. Behavioral observations were collected on all focal subjects using a one-zero sampling technique (Martin and Bateson, 1993) for 17 individual behavioral categories. A behavior was scored if it occurred one or more times during a $1 \mathrm{~min}$ focal subject sample interval. Each session consisted of six $1 \mathrm{~min}$ intervals per focal subject. The 17 behavioral categories were subsequently condensed into 4 composite behavior scores: abnormal, aggression, anxiety, and social behavior. Three different observers were used throughout the study. Initial interobserver reliability tests showed a high correlation $(0.90$ or greater) between different observers. During the Baseline and Post-test periods, the subjects were observed for a total of eight morning (AM) sessions and eight afternoon (PM) sessions. During the Dose periods (Doses 1-8), focal subjects were observed each week for AM and PM sessions during the final injection day ('injection') of the week, usually on Thursday, and on the following no-injection day ('no injection'), usually on Friday. This resulted in AM and PM observation sessions during an 'injection' day and a 'no injection' day for each week of the Dose periods. The AM observations occurred within $60 \mathrm{~min}$ of the first daily dose. PM observations occurred within $60 \mathrm{~min}$ of the last dose of the day (PM observations for Doses 1, 2, and 3 were conducted in the absence of afternoon injection).

Activity monitors. Activity levels were recorded using Actiwatch AW-64 activity monitors (Mini Mitter; Bend, $\mathrm{OR})$. The monitor was attached to the animal's leather identification neck collar and worn continuously for 4-week period. Specific subsets of this massive dataset were averaged for statistical analysis. During each week of the Dose periods, a mean hourly activity count was calculated for each subject across the $4 \mathrm{~h}$ immediately following the last injection of the week (usually a Thursday). Similar

Table I METH Administration: Experimental Design and Dosing Conditions

\begin{tabular}{|c|c|c|c|c|c|c|c|c|c|c|}
\hline Condition & Mon & Tue & Wed & Thu & Fri & $\begin{array}{c}\text { No. of injections } \\
\text { per week }\end{array}$ & $\begin{array}{l}\text { No. injections } \\
\text { per day }\end{array}$ & $\begin{array}{l}\text { No. injections } \\
\text { per condition }\end{array}$ & $\begin{array}{c}\text { Dose } \\
\text { (mg/kg) }\end{array}$ & $\begin{array}{l}\text { No. of } \\
\text { weeks }\end{array}$ \\
\hline Baseline & & & & & & & & & & 4 \\
\hline Dose 2 & & I & & I & & 2 & 1 & 8 & 0.2 & 4 \\
\hline Dose 3 & & 2 & I & 2 & & 5 & I or 2 & 20 & 0.2 & 4 \\
\hline Dose 6 & $2^{\mathrm{a}}$ & 2 & 2 & 2 & & 6 or $8^{a}$ & 2 & 30 & 0.6 & 4 \\
\hline Dose 7 & $3^{\mathrm{a}}$ & 3 & 3 & 3 & & 9 or $12^{\mathrm{a}}$ & 3 & 45 & 0.6 & 4 \\
\hline Dose 8 & $3^{\mathrm{a}}$ & 3 & 3 & 3 & & 9 or $12^{\mathrm{a}}$ & 3 & 57 & 0.6 & 5 \\
\hline Abstinence & & & & & & & & & & 3 \\
\hline
\end{tabular}

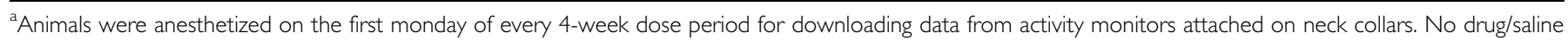


week dose period; the second number refers to the number of injections for weeks 2, 3, and 4 of each dose period. 
hourly activity means were calculated for all subjects across the same $4 \mathrm{~h}$ of the day on the three subsequent 'no injection' days (Friday, Saturday, and Sunday). These data were averaged for each subject across the four 8-week DoseAnalysis periods and used in a three-way repeated-measures analysis of variance (ANOVA) with one between-subjects effect of Treatment (either METH or Control) and two within-subjects effects of Analysis periods and Observation day (injection day-Thursday, Friday, Saturday, and Sunday; see Supplementary Information for details).

Baseline/post-test and dose period behavior comparisons. To simplify the data analysis and to increase the number of behavioral observations per condition, the behavioral data from the eight 4-week Dose periods (Table 1) were collapsed into four 8-week Dose-Analysis periods. All analyses of the behavioral data used two separate repeated measures ANOVA. Behavioral data for the Baseline and Post-test periods were analyzed separately from data for the Dose periods. The first type of ANOVA used one betweensubjects effect (Treatment (either METH or Control)) and one within-subjects effect (Condition (either Baseline or Post-test)). The second type of ANOVA used one betweensubjects effect and two within-subjects effects. The betweensubjects effect was Treatment (same as above). The first within-subjects effect was represented by the four 8-week Dose periods (as Dose 1, 2; Dose 3, 4; Dose 5, 6; and Dose 7, 8). The second within-subjects effect was Observation day (either the 'injection' day or the 'no injection' day of each week). When applicable, mean scores for each dependent variable were calculated for each subject across each of the independent variables. These data were then used as the raw data for all ANOVAs. All data analyses were done on both AM and PM sessions. Preliminary analyses indicated no consistent time of day effects, so all behavioral data were collapsed across AM and PM observational sessions.

Statistical analyses were performed using the SAS programming language (SAS 9.0, Cary, NC) and SPSS (SPSS $11.5)$ and expressed as mean \pm SEM.

METH and AMPH plasma analysis. To obtain blood samples for quantification of plasma METH and AMPH concentrations, animals were anesthetized with ketamine $(8-10 \mathrm{mg} / \mathrm{kg}$, i.m.) at $24 \mathrm{~h}$ following the final METH injection $(0.6 \mathrm{mg} / \mathrm{kg}, \mathrm{i} . \mathrm{m}$.) of the study; $10 \mathrm{~min}$ later, blood samples were taken from the femoral vein, centrifuged, and the plasma fractions were stored at $-80^{\circ} \mathrm{C}$ until analyzed for METH and AMPH by GC-MS.

GC-MS and brain processing procedures. Quantification of METH and AMPH in plasma and the brain tissue processing methods was adapted from our previously published protocols (O'Neil et al, 2006; see Supplementary Information for details).

HPLC procedures. Brain samples were processed and analyzed as previously described (O'Neil et al, 2006; see Supplementary Information for details).

Binding studies, autoradiography, and image analysis. Protocols for the ligand-binding studies $\left(\left[{ }^{3} \mathrm{H}\right]\right.$ WIN35428 (WIN), $\left[{ }^{3} \mathrm{H}\right]$ dihydrotetrabenazine (DTBZ), $\left[{ }^{3} \mathrm{H}\right] \mathrm{SCH} 23390$, and $\left[{ }^{3} \mathrm{H}\right]$ raclopride) and their analysis were adapted from previously reported studies (see Supplementary Information for details). The precommisural striatum that contained the caudate, putamen, and nucleus accumbens was selected for the autoradiography and HPLC studies based on the corresponding coronal image (coronal slice + $13.95 \mathrm{~mm}$ ) of our Internet-based atlas of vervet brain (available at http://labs.pharmacology.ucla.edu/mellab/vervet_atlas/index.html). Contiguous sections were used for the different ligands. The atlas image as identified in our vervet brain atlas and schematic (see Figure 5) show the relative locations of the five circular $(2 \mathrm{~mm}$ diameter) regions of interest (ROIs) for the dorsal caudate, ventral caudate, dorsal putamen, ventral putamen, and nucleus accumbens that were then mapped onto the corresponding digitized autoradiography sections for quantitative analysis. The binding density in each of these ROIs for each animal and each ligand was calculated by subtracting the nonspecific binding from the total binding; the final binding density was then calculated from the mean of triplicate determinations.

Statistics. Repeated measure ANOVAs were performed for analyte content and ligand-binding values in striatal regions using Treatment (METH vs Control) as the betweensubjects factor and brain region (dorsal caudate, ventral caudate, dorsal putamen, ventral putamen, and nucleus accumbens) as the within-subjects factor. Separate ANOVAs were performed for each dependent measure. The following six dependent measures were analyzed separately using the same design: DA, DOPAC, HVA, HVA/DA ratio, 5-HT, and 5-HIAA; $\alpha$ was set at 0.01 .

\section{RESULTS}

\section{Pharmacokinetic Studies}

Values of pharmacokinetic parameters for METH and metabolism-generated AMPH were derived from the single-injection METH study: METH half-life, $4.1 \mathrm{~h}$; AMPH half-life $17.3 \mathrm{~h}$ (Figure 1). Those values were used for model fitting of multiple METH doses (see Supplementary Information for details).

\section{Pharmacokinetic Models}

The pharmacokinetic parameters from METH Study 1 were used to model the time course of weekly plasma concentrations of METH and its metabolite AMPH in the monkey for the final 9 weeks of Dose periods 7 and 8 (see Table 1) that consisted of three daily METH doses of $0.6 \mathrm{mg} / \mathrm{kg}$, at $3 \mathrm{~h}$ intervals for 4 days/week. Since significant behavioral alterations were observed during both METH 'injection' days and 'no injection' days for this period, plasma METH and $\mathrm{AMPH}$ concentrations were measured at $24 \mathrm{~h}$ following the last METH injection of the final week to provide partial validation of the model estimates. Other time points could not be obtained during the METH administration period because the ketamine anesthetic necessary for obtaining blood samples is associated with residual behavioral effects up to $24 \mathrm{~h}$ that would have confounded ongoing behavioral observations (Huff et al, 2003). 

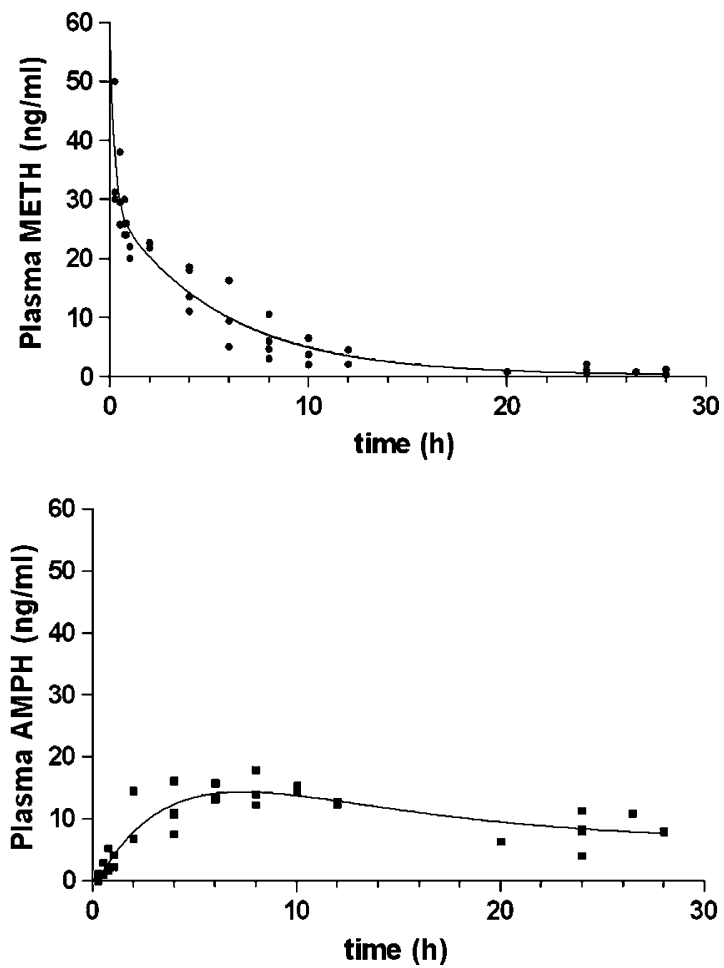

Figure I Plasma concentration vs time curves following METH (0.2 mg/ $\mathrm{kg}$, i.m.) in the vervet monkey $(n=4)$. Upper: plasma METH concentrations (-) from $15 \mathrm{~min}$ to $24-28 \mathrm{~h}$ for each subject; the solid line shows the twocompartment model fitting. Lower: plasma AMPH concentrations at the same time points as analyzed for METH; the solid line shows the one-compartment model fitting.

The pharmacokinetic modeling of the daily METH doses resulted in estimated plasma METH concentrations with Cmax 175-250 $\mu \mathrm{g} / \mathrm{l}(1.2-1.7 \mu \mathrm{M})$ and Cmin 70-100 $\mu \mathrm{g} / \mathrm{l}(0.5-$ $0.7 \mu \mathrm{M})$ between injections. The calculated plasma METH and AMPH concentrations at $24 \mathrm{~h}$ after the last dose of the highest METH administration regimen were $7 \mu \mathrm{g} / 1$ $(0.05 \mu \mathrm{M})$ for METH and $131 \mu \mathrm{g} / \mathrm{l}(\sim 1.0 \mu \mathrm{M})$ for AMPH. At that same time point, the measured plasma concentrations (mean \pm SEM) were $2.7 \pm 1.4 \mu \mathrm{g} / \mathrm{l}(0.02 \mu \mathrm{M})$ for METH and $148 \pm 2.7 \mu \mathrm{g} / \mathrm{l}(1.1 \mu \mathrm{M})$ for AMPH, closely approximating the predicted values (Figure 2). The model also predicted that METH and AMPH had been cleared from the body by $72 \mathrm{~h}$ following the last METH dose of the week.

Since pharmacologically active AMPH concentrations were present throughout these weekly injection periods, a summed plasma AMPH plus METH ('amphetamines') concentration profile was generated for comparison with a human METH 'maintenance' exposure pattern. Based on our recent observations that a significant number of METH abusers had blood METH concentrations in the $1-3 \mu \mathrm{M}$ range (Melega et al, 2007), we calculated that a plasma concentration range similar to the monkey 'amphetamines' profile would be attained by multiple doses of $0.4 \mathrm{mg} / \mathrm{kg}$. Accordingly, the human modeling consisted of a human 'METH maintenance' pattern of three daily doses of $0.4 \mathrm{mg} / \mathrm{kg}$, i.v. at $3 \mathrm{~h}$ intervals for 4 days/week (Figure 2). Both plasma curves showed a Cmax range of 2-3 $\mu \mathrm{M}$, with significant concentrations of drug persisting between injection days, and essentially complete drug clearance by 3 days after the last
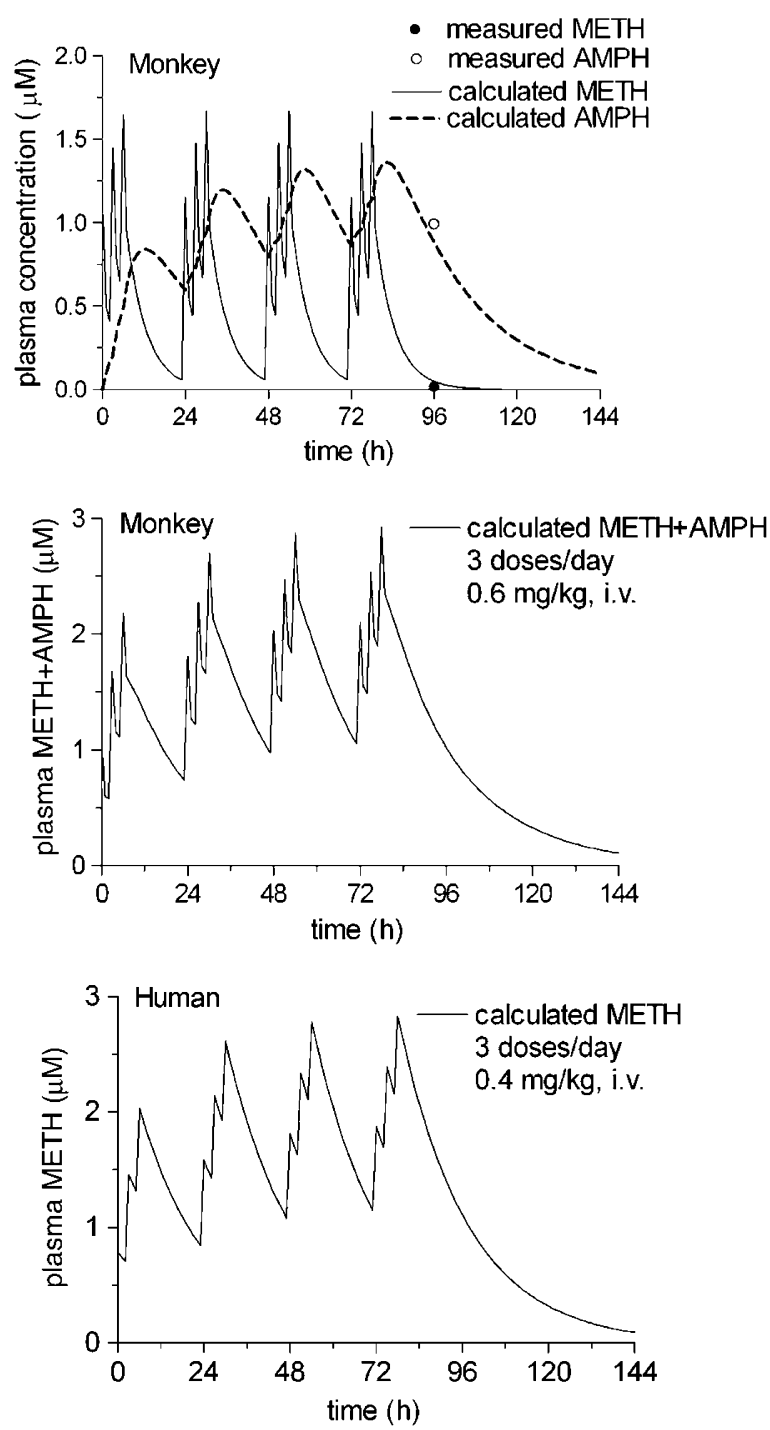

Figure 2 Upper: kinetic modeling of METH) and metabolism-generated plasma AMPH concentrations in the vervet monkey resulting from METH $(0.6 \mathrm{mg} / \mathrm{kg}$, i.v. $)$ administered for 3 doses $/$ day at $3 \mathrm{~h}$ intervals for 4 days. At $24 \mathrm{~h}$ following the last day of this schedule, the calculated plasma concentrations were $7 \mu \mathrm{g} / \mathrm{I}(0.05 \mu \mathrm{M})$ for METH and $\mathrm{I} 3 \mathrm{I} \mu \mathrm{g} / \mathrm{I}(\sim 1.0 \mu \mathrm{M})$ for $\mathrm{AMPH}$. The measured concentrations (mean \pm SEM) in the METH subjects $(n=5)$ were $2.7 \pm \mathrm{I} .4 \mu \mathrm{g} / \mathrm{I}(0.02 \mu \mathrm{M})$ for METH and I $48 \pm 2.7 \mu \mathrm{g} / \mathrm{l}$ (I.I $\mu \mathrm{M}$ ) for AMPH. Middle: kinetic modeling of the summed plasma METH and $\mathrm{AMPH}$ concentrations estimated for the vervet monkey resulting from a METH exposure of 3 doses/day at $3 \mathrm{~h}$ intervals of METH $(0.6 \mathrm{mg} / \mathrm{kg}$, i.v. $)$ for 4 days. Lower: kinetic modeling of plasma METH concentrations estimated for the human resulting from a METH exposure of 3 doses/day at $3 \mathrm{~h}$ intervals of METH $(0.4 \mathrm{mg} / \mathrm{kg}$, i.v. $)$ for 4 days.

exposure of the week. Areas under the curve for drug plasma concentrations $v$ s time were comparable (monkey 'amphetamines' was $87 \%$ that for human METH), suggesting that the 'amphetamines' brain effects from this experimental exposure are relevant for extrapolation to analyzing a corresponding exposure in humans.

\section{Distress Ratings for Capture and Injections}

A lack of aversion to the passive injections over time was suggested from the distress ratings that progressively 

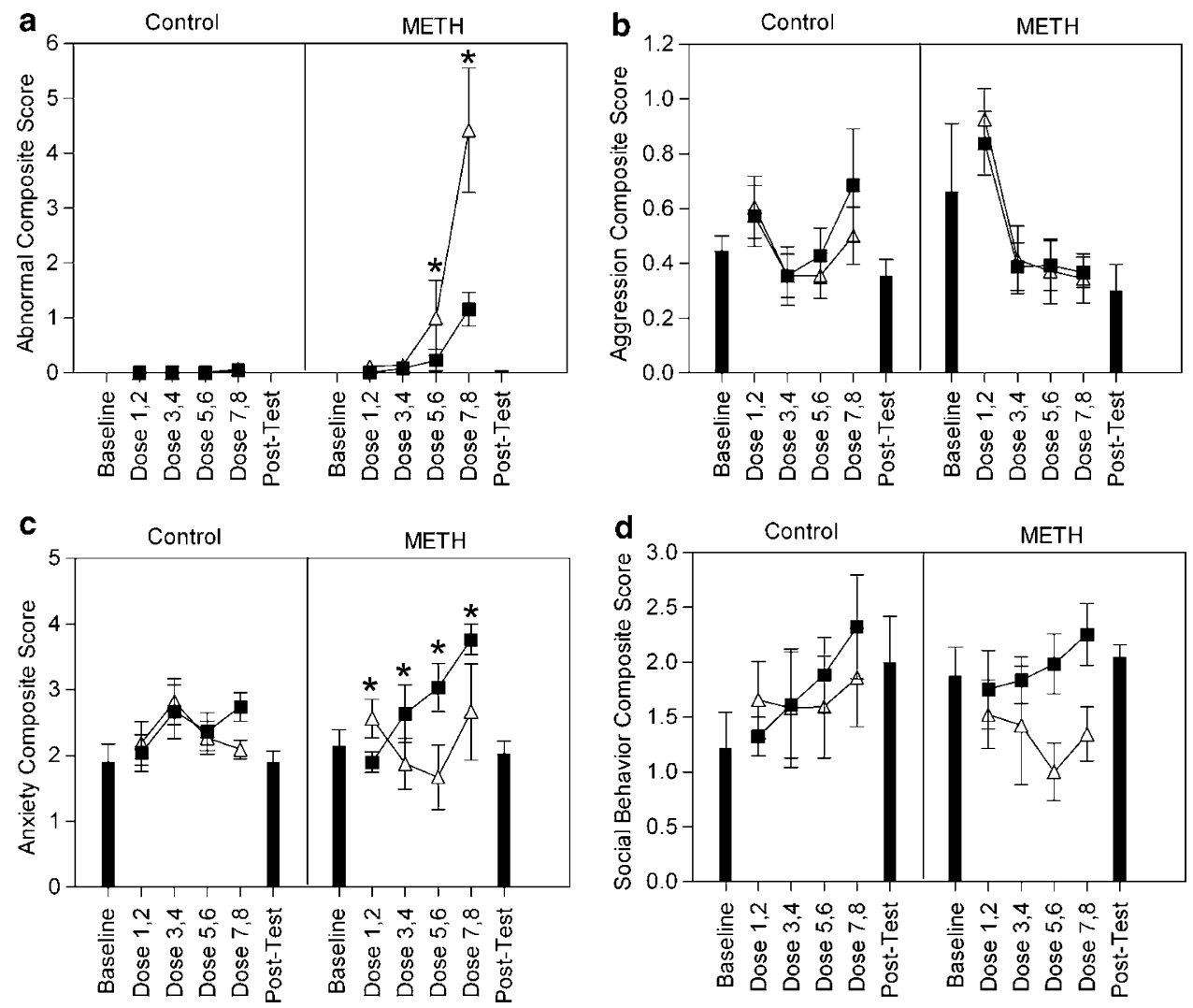

Figure 3 Behavioral composite scores are shown for Control $(n=6)$ and METH $(n=5)$ subjects for the 4-week Baseline and Post-test periods (solid bars). Across the four 8-week periods of drug exposure (see Table I), average behavioral scores (mean \pm SEM) are shown for the last day of the weekly METH or saline injections ( $\Delta$, 'injection') and the following day without injections ( $\mathbf{\square}$, 'no injection'). Significant post hoc comparisons between 'injection' and 'no injection' days are indicated (* $<0.05)$. Results are shown for the dependent behavioral composite scores (a) Abnormal, (b) Aggression, (c) Anxietyrelated, and (d) Social.

decreased throughout the study (see Supplementary Information for details).

\section{Dose Period Comparisons}

The analysis of the dependent variables below shows the behavioral composite scores for the Control and METH subjects across the four Dose Analysis periods (Figure 3, solid lines).

\section{Abnormal Behavior Composite Score}

Dose-dependent increases in species-typical stereotypic actions were observed in the METH subjects (Figure 3a). The Abnormal behavior composite score analysis showed that all main effects, two-way interactions, and the threeway interaction of Treatment $\times$ Analysis Period $\times$ Observation day $(F(3,27)=15.08, p<0.0001)$ were significant. Post hoc tests of simple main effects indicated significantly higher levels of abnormal behavior during 'injection' days compared to 'no injection' days for METH subjects at Dose 5, 6 and Dose 7, $8(p<0.05)$. METH subjects also showed significantly higher levels of abnormal behavior during Dose 7, 8 compared to the other three Dose periods (Dose 1, $2 ; 3,4 ; 5,6 ; p<0.05$ ). This increase was significant for both 'injection' days as well as for 'no injection' days. In addition,
METH subjects showed significantly higher levels of abnormal behavior compared to Controls only during Dose $7,8(p<0.05)$. This difference was also significant for both 'injection' days as well as for 'no injection' days.

\section{Aggression Composite Score}

Levels of aggression changed across Dose periods for both Control and METH subjects (Figure $3 \mathrm{~b}$ ). Analysis of the Aggression composite score showed a significant effect of Dose-Analysis period $(\mathrm{F}(3,27)=8.83, p<0.001)$ and a significant Treatment $\times$ Analysis period interaction $(\mathrm{F}(3,27)=3.74, p<0.05)$. For Controls, levels of aggression were significantly higher during the time period of Dose 7, 8 than at Dose 3, 4. For METH subjects, aggression was significantly higher at Dose 1,2 compared to Dose 3, 4 and Dose 5, 6 .

\section{Anxiety Composite Score}

Increases in the anxiety scores were observed in the METH subjects for most Dose-Analysis periods during 'no injection' days when AMPH but not METH was present in pharmacologically active concentrations (Figure 3c). The Anxiety composite score analysis showed a significant three-way interaction of Treatment $\times$ Dose-Analysis 
period $\times$ Observation day $(\mathrm{F}(3,27)=6.70, p<0.01)$. Tests of simple main effects indicated METH subjects showed significantly lower levels of anxiety-related behavior during 'no injection' days compared to 'injection' days for Dose 1, 2 , but showed significantly higher levels of anxiety during 'no injection' days compared to 'injection' days for Dose 3, 4; Dose 5, 6; and Dose 7, $8(p<0.05)$. In addition, METH subjects during 'no injection' days showed significantly higher levels of anxiety-related behavior during Dose 5, 6 and Dose 7, 8 compared to Dose 1, $2(p<0.05)$. Control subjects also showed higher levels of anxiety-related behavior during Dose 7, 8 compared to Dose 1, 2 during 'no injection' days $(p<0.05)$.

\section{Social Behavior Composite Score}

The METH subjects generally showed lower social behavior on 'injection' days, ie following METH administration (Figure 3d). Analysis of the social composite scores showed a significant Treatment $\times$ Dose-Analysis period interaction $(\mathrm{F}(1,9)=8.18, p<0.05)$. Control subjects showed relatively similar levels of social interaction during 'injection' and 'no injection' time periods, while METH subjects showed significantly lower levels of social behavior on 'injection' days $(p<0.05)$. There was also a significant Treatment $\times$ Dose-Analysis period interaction $(\mathrm{F}(3,27)=4.24$, $p<0.05)$. For both METH and Control subjects, levels of social behavior were significantly higher on 'no injection' days compared to 'injection' days for Dose 5, 6 and Dose 7, $8(p<0.05)$.

\section{Baseline/Post-Test Behavior Comparisons}

No significant differences were found between the Control and METH subjects across the Baseline and Post-test periods (Figure 3, indicated by the solid bars for each behavior). Results indicated no significant main effects for Treatment or Condition, and no significant interactions for the Abnormal, Social, or Anxiety composite behavior scores. Levels of Aggression tended to decrease between Baseline and Post-test periods for both sets of subjects, but this reduction was not significant $(\mathrm{F}(1,9)=3.69, p=0.087)$. There were no significant changes between pre- and poststudy weights for either group (see Supplementary Information for details).

\section{Activity Monitor Data}

Activity changes were analyzed for $4 \mathrm{~h}$ time periods following the last injection of the week on Thursday and for the same time periods on the following Friday, Saturday, and Sunday. Increases in activity changes were observed in the METH subjects for Dose period 5, 6 and more so for period 7, 8 when the METH doses were highest. The repeated-measures ANOVA showed a significant threeway interaction of Treatment $\times$ Dose-Analysis period $\times$ Observation day $(\mathrm{F}(9,81)=3.14, p<0.01)$. Post hoc tests of simple main effects indicated significantly higher activity counts for the METH subjects compared to Controls (Figure 4) during Dose 5, 6 on Fridays, and during Dose 7,8 on Thursday - injection days and the following Fridays $(p<0.05)$. No significant differences between METH and

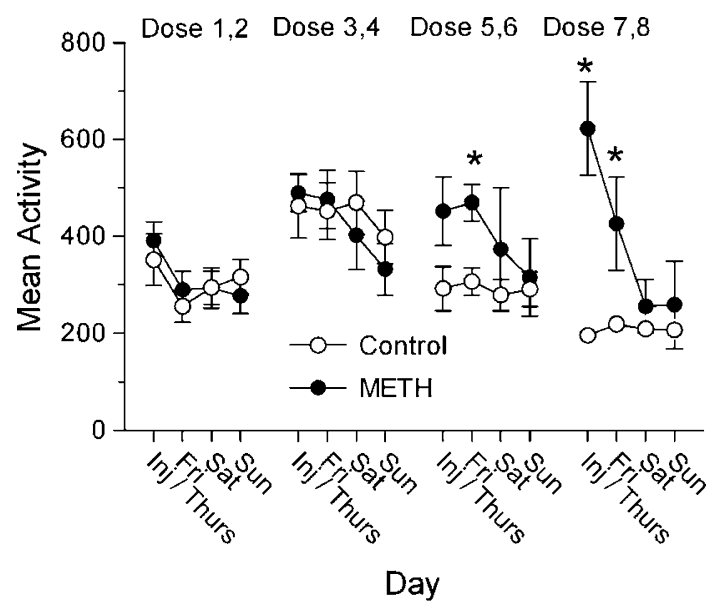

Figure 4 Activity levels (mean \pm SEM) were obtained with activity monitors and are shown for the $4 \mathrm{~h}$ period following the final 'injection day' of each week (Thurs), and for the same time periods on the subsequent three 'no injection' days. METH subjects $(n=5)$ showed higher activity levels than Controls $(n=6)$ only during later dose periods (Dose 5, 6 and Dose 7,8 ) as measured for the $4 \mathrm{~h}$ period on the final 'injection day,' and for the same time period on the following 'no injection' Friday $(* p<0.05)$.

Control subjects were found across any other Dose period/ Observation day periods.

\section{Striatal Dopamine and Serotonin Content}

In the $5 \mathrm{~mm}$ block of brain tissues contralateral to those used for the ligand-binding studies, regions of caudate, putamen, and nucleus accumbens (Figure 5) were dissected for quantitation of DA and 5-HT and their respective metabolites by HPLC-ED. The METH subjects had approximately $20 \%$ lower DA content, $11.2 \pm 0.5 \mu \mathrm{g} / \mathrm{g}$ wet weight, compared to controls, $13.8 \pm 0.5 \mu \mathrm{g} / \mathrm{g}$ (mean \pm SEM across all five regions). Analysis of DA content indicated a significant effect of condition $(\mathrm{F}(1,9)=13.70, p<0.005)$, and a significant effect of brain region $(\mathrm{F}(3,27)=61.21, p<0.0001)$, but no significant interaction $(\mathrm{F}(3,27)=1.76, \mathrm{NS})$.

Analysis of the DOPAC-, HVA-, 5-HT-, and 5-HIAAdependent measures all yielded significant effects of region (DOPAC: $\mathrm{F}(3,27)=16.47, p<0.0001$; $\operatorname{HVA}: \mathrm{F}(3,27)=32.42$, $p<0.0001$; 5 -HT: $\quad \mathrm{F}(3,27)=7.28, \quad p<0.001$; 5 -HIAA: $\mathrm{F}(3,27)=43.59, p<0.0001$ ) (see Supplementary Information for 5-HT, and 5-HIAA $\mu$ g/g wet weight values). For all four of these dependent measures there were no significant effects of condition and no significant condition $\times$ region interactions. HVA/DA ratios for the METH subjects were approximately $30 \%$ higher than controls. The analysis showed a significant effect of condition $(\mathrm{F}(1,9)=35.93, p<0.001)$ and a significant effect of region $(\mathrm{F}(3,27)=54.38, p<0.0001)$ but no significant interaction $(\mathrm{F}(3,27)=2.11, \mathrm{NS})$.

\section{$\left[{ }^{3} \mathrm{H}\right]$ WIN35428}

WIN binding differed across striatal brain regions and was significantly lower in the METH animals across all five regions analyzed (Figure 6, upper part): METH 28.6 \pm 1.4 , Control $45.6 \pm 1.8 \mathrm{fmol} / \mathrm{mg}$ protein (mean $\pm \mathrm{SEM}$ ). WIN binding showed a significant effect of condition $(\mathrm{F}(1,9)=35.97, p<0.001)$ and significant effect of region 

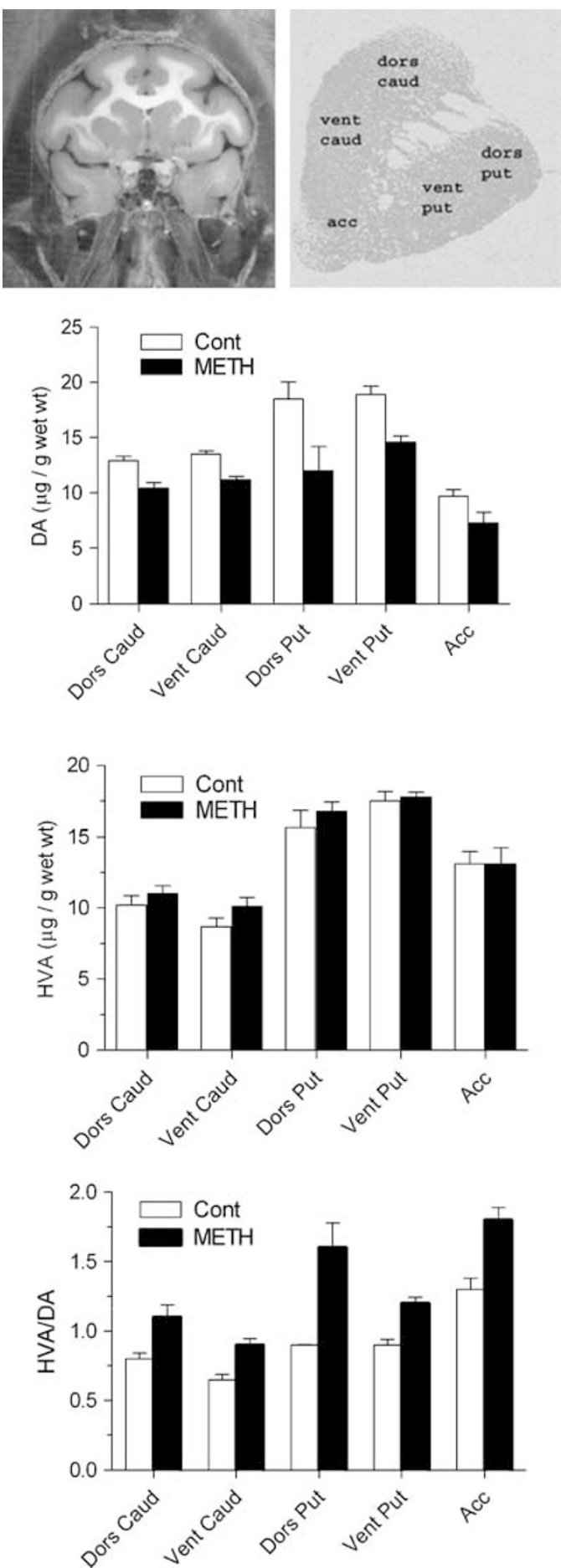

Figure 5 Upper left: coronal image of the cryosectioned vervet brain showing the precommissural striatum corresponding to the region used for autoradiography studies. Upper right: analysis-ROI labeled over representative areas containing dorsal caudate (Dors Caud), ventral caudate (Vent Caud), dorsal putamen (Dors Put), ventral putamen (Vent Put), and nucleus accumbens (Acc). DA and HVA content in regions of precommissural striatum following an 8-month escalating dose-METH exposure and 3 weeks of abstinence in METH subjects $(n=5)$ and control $(n=6)$ subjects. Upper: DA content; Middle, HVA content; Lower, HVA/ DA ratios. For the METH subjects, DA was significantly lower (20\%) and HVA/DA significantly higher (30\%) across the five striatal regions relative to controls $(p<0.05)$.
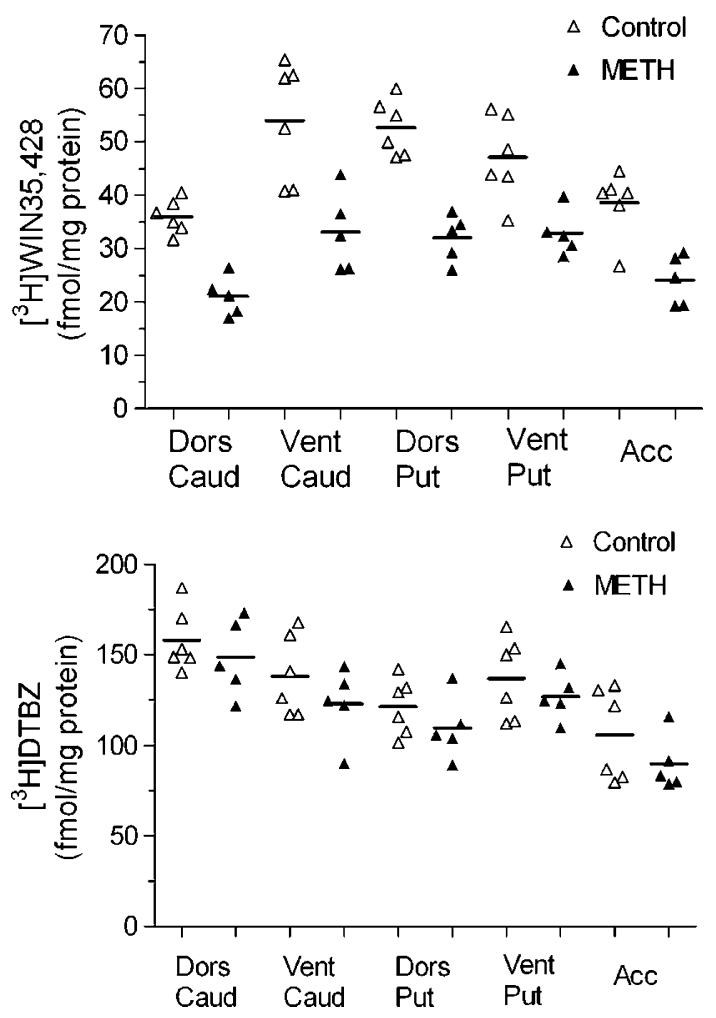

Figure 6 Autoradiography results of presynaptic transporter ligand binding in coronal sections of precommissural striatum in METH $(n=5)$ and Control $(n=6)$ subjects at 3 weeks after the 8-month escalating doseMETH exposure. Upper: $\left.{ }^{3} \mathrm{H}\right] \mathrm{WIN} 35428$ (WIN) binding to the dopamine reuptake transporter; $\mathrm{METH}$ subjects showed significantly lower binding across the five striatal regions relative to controls $(p<0.05)$. Lower: $\left[{ }^{3} \mathrm{H}\right]$ dihydrotetrabenazine (DTBZ) binding to the vesicular monoamine transporter. Across the five striatal regions, no significant differences were detected between Control and METH subjects in ligand binding (METH-lower by 10\%).

$(\mathrm{F}(4,36)=22.09, p<0.001)$, but showed no significant interaction $(\mathrm{F}(4,36)=1.43$, NS).

\section{$\left[{ }^{3} \mathrm{H}\right]$ Dihydrotetrabenazine}

DTBZ binding differed across striatal brain regions (Figure 6, lower part). The mean 10\% lower DTBZ binding for the METH subjects was not significantly different from Controls: control $132.0 \pm 4.8$ vs METH $119.5 \pm 5.1 \mathrm{fmol} / \mathrm{mg}$ protein (mean \pm SEM across all five regions). The DTBZ analysis showed no effect of condition $(\mathrm{F}(1,9)=1.33$, NS), a significant effect of region $(\mathrm{F}(4,36)=58.19, p<0.0001)$, and no significant interaction $(F(4,36)=0.30, \mathrm{NS})$.

\section{$\left[{ }^{3} \mathrm{H}\right] \mathrm{SCH} 23390$}

The ED-METH exposure did not alter $\left[{ }^{3} \mathrm{H}\right] \mathrm{SCH} 23390$ binding in striatal brain regions: control 25.8. $\pm 0.8 \mathrm{vs}$ METH $27.8 \pm 0.8 \mathrm{fmol} / \mathrm{mg}$ protein (mean \pm SEM across all five regions). The analysis showed a significant main effect of region $(\mathrm{F}(4,36)=16.58, p<0.0001)$ but no significant main effect of condition $(F(1,9)=0.24$, NS). The condition $\times$ region interaction was nearly significant $(\mathrm{F}(4,26)=2.43, p=0.066)$. 


\section{$\left[{ }^{3} \mathrm{H}\right]$ Raclopride}

The ED-METH exposure did not alter $\left[{ }^{3} \mathrm{H}\right]$ raclopride binding in striatal brain regions: Control $66.0 \pm 1.0$ vs $\mathrm{METH} 67.1 \pm 1.4 \mathrm{fmol} / \mathrm{mg}$ protein (mean \pm SEM across all five regions). The analysis showed a significant main effect of region $(\mathrm{F}(4,36)=36.26, p<0.0001)$ but no significant main effect of condition $(\mathrm{F}(1,9)=0.34$, NS) and no significant interaction $(\mathrm{F}(4,36)=1.24$, NS) (see Supplementary Information for $\left[{ }^{3} \mathrm{H}\right] \mathrm{SCH} 23390$ and $\left[{ }^{3} \mathrm{H}\right]$ raclopride details).

\section{DISCUSSION}

The aim of this study was to establish in socially housed vervet monkeys a profile of behavioral and brain alterations resulting from modeling an ED-METH exposure extending over 8 months. The principal observations were dosedependent increases in abnormal and anxiety-related behaviors, activity levels, and decreases in aggression. None of those behavioral changes, however, were observed during a subsequent 3 weeks of drug abstinence despite 35\% lower striatal DAT ligand binding and 20\% lower DA content measured after that observation period. The brain alterations resulting from this low to moderate METH exposure with peak blood concentrations less than $3 \mu \mathrm{M}$ were not characteristic of extensive neurodegeneration, but did suggest a relatively low threshold for METH-induced persistent effects on presynaptic striatal DA system integrity.

\section{Pharmacokinetic Modeling}

We addressed issues not frequently included in nonhuman primate METH studies, eg species-specific pharmacokinetic modeling coupled with escalating doses/frequency of administration to group-housed subjects in sufficient numbers for both behavioral and brain statistical analyses. Recognizing that no one experimental protocol can model all aspects of human METH exposure patterns, we used an ED-METH protocol to model some of the predominant features that frequently accompany drug abuse, namely, a gradual increase in dose and frequency of administration. Yet, caveats remain. For example the periodic increases in the experimental dosing regimen did not parallel the inconsistent METH intake quantities associated with most human abuse patterns. However, periodic use has also been reported in human METH studies, with majority patterns characterized by 2-3 injections/day for 1-2 days/week (Miyazaki, 1986; Zule and Desmond, 1999), and 1-3 times /day for 3-20 days/month (Simon et al, 2002)-METH frequency ranges that did correspond to our experimental protocol. Although the METH doses we modeled were less than the daily $0.5-1 \mathrm{~g}$ quantities self-reported in the human study by Simon et al, those amounts may represent overestimates since they were not independently verified for either METH quantity or drug purity. Nonetheless, the estimated peak plasma METH concentrations of $1-3 \mu \mathrm{M}$ during the past 2 months of this experimental exposure did correspond to plasma METH concentrations measured in several studies of human abusers (Miyazaki, 1986; Logan et al, 1998; Melega et al, 2007).
Lastly, since the passive administration of METH by i.m. injections was not intended to model the i.v. or smoking routes of self-administration and its associated acute behavioral changes, the behavioral effects we observed were interpreted as a function of cumulative plasma METH and $\mathrm{AMPH}$ concentrations as achieved throughout this study. Accordingly, the time frame for the emergence of behavioral alterations consequent to those METH exposures did represent conditions that paralleled some aspects of human abuse, eg emergence of abnormal behaviors at higher doses.

\section{Behavioral Alterations}

During the first 2 months of infrequent exposure (1-2 doses/week), METH-induced changes in the behavioral composite scores were minimal but became apparent with multiple doses, and further increased when dose quantity and administration frequency escalated to 2-3 daily doses for 4 days/week for Dose periods 5, 6 and 7, 8 .

The plasma METH and AMPH concentration profiles as calculated for those two final analysis periods provided a pharmacokinetic model for assessing behavioral changes during both METH 'injection' and 'no injection' days. For example, during Dose 5, 6 and 7, 8, the Abnormal behaviors were increased on 'injection' days and then markedly decreased on the subsequent 'no injection' days. However, at those times, plasma AMPH concentrations (Figure 2) were still in a pharmacologically active range $(132 \mu \mathrm{g} / \mathrm{l}$, $\sim 1 \mu \mathrm{M}$ ), indicating significant pharmacological tolerance in the presence of AMPH. Although the pharmacodynamic effects of AMPH were not assessed independently in this study, prior studies have established, in vivo, that METH and AMPH are equipotent for increasing extracellular DA concentrations in rodent striatum (Kuczenski et al, 1995; Melega et al, 1995). Thus, the lack of efficacy by AMPH to sustain the abnormal behaviors may be due to either depletion of DA content and/or adaptations associated with lower DAT availability.

However, this observation of behavioral tolerance in the presence of pharmacologically active plasma AMPH concentrations was not apparent in the analysis of the other behaviors. Anxiety measures of the METH subjects increased on 'no injection' days from Dose 3, 4 to 7,8 , while the Aggression composite scores showed decreases on both 'injection' and 'no injection' days, not paralleling either the pattern of Abnormal or Anxiety measures. It is likely that expression of those behaviors was a function of the pharmacological actions of METH and AMPH and subsequent complex behavioral interactions within the social groups. For example, METH and AMPH exposure in both clinical and experimental studies have often been associated with increases in the rates of aggression, however, we observed consistent decreases in the METH subjects between Dose periods 3, 4 and 7, 8. Here, the extent of AMPHs-associated aggression was likely affected by the overall dynamics of the social environment that constrained rather than provoked its expression (Miczek and Gold, 1983; King and Ellinwood, 1992).

For all Dose periods, the housing of control and METH animals in the same social setting for longitudinal behavioral assessments showed the necessity of having a 
control group rather than a within-subject study design. For example, increases observed in affiliative social behavior were time dependent, irrespective of METH treatment. Those increases likely resulted from the continuing habituation of the group throughout the 8 months of drug exposure, and also may have offset negative group interactions insofar as drug-induced aggression increases did not materialize during any Dose period. Yet, METHdependent effects could still be distinguished in the social group environment as evidenced by increases in activity only in the METH subjects following their last dose of the week while Control subjects' activity remained unchanged over the same time period.

Overall, we conclude that the rather limited expression of the METH-associated behavioral effects observed in this study was related to the species-typical social environment provided by the group housing. Nonetheless, the timedependent changes in Abnormal and Anxiety behaviors that could be reliably assessed on 'injection' and 'no injection' days provide an experimental platform for the evaluation of pharmacological interventions. This social model may represent a more realistic setting to assess drug-induced behaviors than in animals evaluated in isolated conditions. Housing condition as an experimental variable has been studied across a range of monkey species and those results have consistently shown that measures of behavioral indices (eg stereotypy and abnormal behavior), are reduced in socially housed animals as compared to those individually housed (Gust et al., 1993; Lutz et al., 2003). For comparison, METH and AMPH dosages similar to that administered in our study resulted in more prolonged and extreme behavioral alterations in male-only groups (Machiyama, 1992) and in singly-housed animals (Castner and GoldmanRakic, 1999).

\section{Brain Alterations}

Subregions of precommissural striatum were evaluated to establish a potential heterogeneity of deficits (putamen $>$ caudate $>>$ nucleus accumbens regions) as we had observed in prior studies for DA content and immunoreactivity of DA system markers (TH, DAT, and VMAT) with acute METH exposure of two doses of $2 \mathrm{mg} / \mathrm{kg}$, i.m., $24 \mathrm{~h}$ apart (Harvey et al, 2000). That dosage resulted in significantly greater decreases in both DAT $(75-90 \%)$ and VMAT (40-60\%) measures whereas only moderate decreases in DAT (35\%) and VMAT (10\%, NS) ligand binding resulted from the present ED-METH exposure. The apparent greater sensitivity of the DAT to METH's actions was consistent with prior observations following ED-METH exposure in rodents (Segal et al, 2003, 2005). Since parallel losses of both DAT and VMAT binding have been used as indirect evidence for structural loss of DA terminals (Frey et al, 1997), the finding of predominantly lower DAT binding suggested that neuronal loss/axonal degeneration was not a significant component of the neurotoxicity profile. These findings parallel the lower striatal DAT ligand binding (30-50\%) without significant changes in VMAT (Wilson et al, 1996), observed in a post-mortem human study conducted on individuals whose final METH exposure (plasma METH concentrations, range $12-84 \mu \mathrm{M}$ ) was associated with their imminent death.
Likewise, the DAT ligand-binding results in this monkey study were similar to those obtained from PET imaging of abstinent human METH abusers. After several months of abstinence, those individuals showed 15-30\% lower striatal DAT ligand binding. The changes in the DAT were rather limited as suggested by the extensive overlap of individual values shown in the scatter plots of METH and control groups (Volkow et al, 2001; Johanson et al, 2006). Similarly, a recent PET imaging study that assessed both DAT and VMAT ligand binding in METH-dependent individuals reported lower values of only 15 and $10 \%$, respectively, compared to controls (Johanson et al, 2006). Since only subjects who met DSM-IV criteria for METH dependence (ie not infrequent or short-term abusers) were imaged in these studies, it is not likely that individuals with a greater magnitude or duration of exposure will be characterized, in vivo, in the future. Thus, it appears that METHassociated decreases in DAT ligand binding with minimal or no changes for VMAT are observed in both human and animal studies and future research should be directed to determining the functional significance of that profile of striatal DA system alterations.

Although comorbid psychiatric disorders as well as ongoing or prior use of other drugs (eg nicotine and alcohol) may be contributing factors that further distinguish human METH brain profiles, our animal model results demonstrated that those factors were not necessary concomitants of METH-induced presynaptic striatal DA system deficits, as indicated by the $20 \%$ lower DA content and $35 \%$ lower DAT binding. The absence of changes in other measured parameters, eg DA D1 and D2 receptor densities, suggested that a higher cumulative dosage than administered in this study may be necessary for such METH-induced alterations. However, the few human studies that have assessed DA D2 receptors in METH abusers have not shown marked decreases in these parameters. For example, in $\left[{ }^{11} \mathrm{C}\right]$ raclopride-PET studies, decreases in DA D2 receptor availability of only $10 \%$ in putamen and $16 \%$ in caudate were measured (Volkow et al, 2001) while a post-mortem study showed a trend of $25 \%$ decreases in D2 receptor protein levels (Worsley et al, 2000). The range of cumulative METH exposure patterns for those individuals may have contributed to the variability of those measures. We hypothesize that with our escalating dose-METH model, exposure to higher peak plasma METH concentrations than achieved in the present study will result in DA receptor density alterations.

Although the striatum was the focus of biochemical analysis for this study, METH's actions also impact biogenic amine systems throughout cortical and limbic brain regions. METH-induced pharmacologic effects within those systems also likely contributed to the observed changes in behavior. However, given the relatively small magnitude of striatal DA and DAT alterations in this study, detection of biochemical changes in other regions with sparser DA and 5-HT innervation was not apparent and may only be associated with greater METH exposure.

In conclusion, this METH administration protocol in the monkey modeled some aspects of a human daily multiple dose exposure and caused significant behavioral alterations in categories relevant to the human METH condition, eg anxiety, abnormal behavior, that were likely the result of 
alterations in both dopaminergic and nondopaminergic systems. The absence of changes in the monitored behaviors during the METH abstinence period could signify that the reductions in the striatal DA and DAT measures were not of sufficient magnitude to effect changes in those behaviors, or that they were not relevant to their expression. Conversely, we propose that other biologic stressors, either pharmacologically or environmentally induced, acting on a compromised DA system and downstream circuits will produce METH-related behavioral effects in the absence of drug. Identification of those factors would suggest targeted pharmacotherapy for the treatment of behavioral disorders associated with long-term METH exposure. For addressing public health concerns related to METH abuse, the present results show that an escalating dose METH exposure, not associated with acute high-dose binges, is sufficient to produce presynaptic striatal DA system alterations that persist at least 3 weeks after last exposure.

\section{ACKNOWLEDGEMENTS}

This work was supported by Grant DA 11237 from the National Institute of Mental Health (to WPM).

\section{DISCLOSURE/CONFLICT OF INTEREST}

There are no potential conflicts of interest for any of the authors as it relates to the subject of the report. None of the authors have received compensation for professional services from organizations, companies, or individuals in any of the previous 3 years nor do they anticipate receiving such compensations in the near future.

\section{REFERENCES}

Bowyer JF, Holson RR (1995). Methamphetamine and amphetamine neurotoxicity. In: Chang LW, Dyers RS (eds). Handbook of Neurotoxicology. Marcel Dekker: New York. pp 845-870.

Cadet JL, Jayanthi S, Deng X (2003). Speed kills: cellular and molecular bases of methamphetamine-induced nerve terminal degeneration and neuronal apoptosis. FASEB J 17: 1775-1788.

Castner SA, Goldman-Rakic PS (1999). Long-lasting psychotomimetic consequences of repeated low-dose amphetamine exposure in rhesus monkeys. Neuropsychopharmacology 20: 10-28.

Cho AK, Melega WP, Kuczenski R, Segal DS (2001). Relevance of pharmacokinetic parameters in animal models of methamphetamine abuse. Synapse 39: 161-166.

Cook CE, Jeffcoat AR, Hill JM, Pugh DE, Patetta PK, Sadler BM et al (1993). Pharmacokinetics of methamphetamine selfadministered to human subjects by smoking hydrochloride. Drug Metab Dispos 21: 717-723.

Cretzmeyer M, Sarrazin MV, Huber DL, Block RI, Hall JA (2003). Treatment of methamphetamine abuse: research findings and clinical directions. J Subst Abuse Treat 24: 267-277.

Davidson C, Gow AJ, Lee TH, Ellinwood EH (2001). Methamphetamine neurotoxicity: necrotic and apoptotic mechanisms and relevance to human abuse and treatment. Brain Res Rev 36: 1-22.

Fischman MW, Schuster CR (1977). Long-term behavioral changes in the rhesus monkey after multiple daily injections of D-methylamphetamine. J Pharmacol Exp Ther 201: 593-605.

Frey K, Kilbourn M, Robinson T (1997). Reduced striatal vesicular monoamine transporters after neurotoxic but not after behaviorally-sensitizing doses of methamphetamine. Eur J Pharmacol 334: 273-279.
Gust DA, Gordon TP, Hambright MK (1993). Response to removal from and return to a social group in adult male rhesus monkeys. Physiol Behav 53: 599-602.

Harvey DC, Lacan G, Melega WP (2000). Regional heterogeneity of dopaminergic deficits in vervet monkey striatum and substantia nigra after methamphetamine exposure. Exp Brain Res 133: 349-358.

Huff A, Jorgensen M, Melega WP, Fairbanks LA (2003). The effects of ketamine on vervet monkeys. Am J Primatology 60: 92-93.

Johanson CE, Frey KA, Lundahl LH, Keenan P, Lockhart N, Roll J et al (2006). Cognitive function and nigrostriatal markers in abstinent methamphetamine abusers. Psychopharmacology (Berl) 185: 327-338.

King G, Ellinwood Jr E (1992). Amphetamines and other stimulants. In: Lowinson J, Ruiz P, Millman R and Langrod J (eds). Substance Abuse: A Comprehensive Textbook. Williams \& Wilkins: Baltimore. pp 247-270.

Kuczenski R, Segal D, Cho A, Melega W (1995). Hippocampus norepinephrine, caudate dopamine and serotonin, and behavioral responses to the stereoisomers of amphetamine and methamphetamine. J Neurosci 15: 1308-1317.

Lineberry TW, Bostwick JM (2006). Methamphetamine abuse: a perfect storm of complications. Mayo Clin Proc 81: 77-84.

Ling W, Rawson R, Shoptaw S (2006). Management of methamphetamine abuse and dependence. Curr Psychiatry Rep 8: 345-354.

Logan BK, Fligner CL, Haddix T (1998). Cause and manner of death in fatalities involving methamphetamine. J Forensic Sci 43: 28-34.

Lutz C, Well A, Novak M (2003). Stereotypic and self-injurious behavior in rhesus macaques: a survey and retrospective analysis of environment and early experience. Am J Primatol 60: $1-15$.

Machiyama Y (1992). Chronic methamphetamine intoxication model of schizophrenia in animals. Schizophr Bull 18: 107-113.

Madden LJ, Flynn CT, Zandonatti MA, May M, Parsons LH, Katner SN et al (2005). Modeling human methamphetamine exposure in nonhuman primates: chronic dosing in the rhesus macaque leads to behavioral and physiological abnormalities. Neuropsychopharmacology 30: 350-359.

Martin P, Bateson P (1993). Measuring Behaviour: An Introductory Guide. Cambridge University Press: Cambridge.

McCann UD, Wong DF, Yokoi F, Villemagne V, Dannals RF, Ricaurte GA (1998). Reduced striatal dopamine transporter density in abstinent methamphetamine and methcathinone users: evidence from positron emission tomography studies with [C-11]WIN-35, 428. J Neurosci 18: 8417-8422.

Melega WP, Cho AK, Harvey D, Lacan G (2007). Methamphetamine blood concentrations in human abusers: application to pharmacokinetic modeling. Synapse 61: 216-220.

Melega WP, Raleigh MJ, Stout DB, Lacan G, Huang SC, Phelps ME (1997). Recovery of striatal dopamine function after acute amphetamine- and methamphetamine-induced neurotoxicity in the vervet monkey. Brain Res 766: 113-120.

Melega WP, Williams AE, Schmitz D, Stefano ED, Cho AK (1995). Pharmacokinetic and pharmacodynamic analysis of the actions of $\mathrm{D}$-amphetamine and $\mathrm{D}$-methamphetamine on the dopamine terminal. J Pharmacol Exp Ther 274: 90-96.

Meredith CW, Jaffe C, Ang-Lee K, Saxon AJ (2005). Implications of chronic methamphetamine use: a literature review. Harv Rev Psychiatry 13: 141-154.

Miczek KA, Gold LH (1983). -amphetamine in squirrel monkeys of different social status: effects on social and agonistic behavior, locomotion, and stereotypies. Psychopharmacology 81: $183-190$.

Miyazaki T (1986). Relation between methamphetamine and amphetamine concentrations in the blood and symptoms in methamphetamine abusers. Nihon Hoigaku Zasshi 40: 132-139. 
O’Neil ML, Kuczenski R, Segal DS, Cho AK, Lacan G, Melega WP (2006). Escalating dose pretreatment induces pharmacodynamic and not pharmacokinetic tolerance to a subsequent high-dose methamphetamine binge. Synapse 60: 465-473.

Segal DS, Kuczenski R, O’Neil ML, Melega WP, Cho AK (2003). Escalating dose methamphetamine pretreatment alters the behavioral and neurochemical profiles associated with exposure to a high-dose methamphetamine binge. Neuropsychopharmacology 28: 1730-1740.

Segal DS, Kuczenski R, O’Neil ML, Melega WP, Cho AK (2005). Prolonged exposure of rats to intravenous methamphetamine: behavioral and neurochemical characterization. Psychopharmacology (Berl) 180: 501-512.

Seiden LS (1985). Methamphetamine: toxicity to dopaminergic neurons. NIDA Res Monogr 62: 100-116.

Seiden LS, Sabol KE (1996). Methamphetamine and methylenedioxymethamphetamine neurotoxicity: possible mechanisms of cell destruction. NIDA Res Monogr 163: 251-276.

Sekine Y, Iyo M, Ouchi Y, Matsunaga T, Tsukada H, Okada $\mathrm{H}$ et al (2001). Methamphetamine-related psychiatric symptoms and reduced brain dopamine transporters studied with PET. Am J Psychiatry 158: 1206-1214.
Simon SL, Richardson K, Dacey J, Glynn S, Domier CP, Rawson R et al (2002). A comparison of patterns of methamphetamine and cocaine use. J Addict Dis 21: 35-44.

Volkow ND, Chang L, Wang GJ, Fowler JS, Leonido-Yee M, Franceschi D et al (2001). Association of dopamine transporter reduction with psychomotor impairment in methamphetamine abusers. Am J Psychiatry 158: 377-382.

Wang GJ, Volkow ND, Chang L, Miller E, Sedler M, Hitzemann R et al (2004). Partial recovery of brain metabolism in methamphetamine abusers after protracted abstinence. Am J Psychiatry 161: 242-248.

Wilson JM, Kalasinsky KS, Levey AI, Bergeron C, Reiber G, Anthony RM et al (1996). Striatal dopamine nerve terminal markers in human, chronic methamphetamine users. Nature Medicine 2: 699-703.

Worsley JN, Moszczynska A, Falardeau P, Kalasinsky KS, Schmunk $\mathrm{G}$, Guttman $\mathrm{M}$ et al (2000). Dopamine D1 receptor protein is elevated in nucleus accumbens of human, chronic methamphetamine users. Mol Psychiatry 5: 664-672.

Zule WA, Desmond DP (1999). An ethnographic comparison of HIV risk behaviors among heroin and methamphetamine injectors. Am J Drug Alcohol Abuse 25: 1-23.

Supplementary Information accompanies the paper on the Neuropsychopharmacology website (http://www.nature.com/npp) 